\title{
Synchronous Breast and Rectal Cancers in a Man
}

\author{
Anastasios J. Karayiannakis ${ }^{a}$ Stylianos Kakolyris ${ }^{b}$ \\ Georgios Kouklakis ${ }^{c}$ Leonidas Chelis ${ }^{b}$ Helen Bolanaki ${ }^{a}$ \\ Christos Tsalikidis $^{\mathrm{a}}$ Constantinos Simopoulos $^{\mathrm{a}}$ \\ a Second Department of Surgery, bepartment of Clinical Oncology and \\ 'Gastrointestinal Endoscopy Unit, Democritus University of Thrace, Medical \\ School, Alexandroupolis, Greece
}

\section{Key Words}

Colorectal carcinoma - Male breast cancer $\cdot$ Second primary cancer $\cdot$ Synchronous cancer

\begin{abstract}
Breast cancer in men is relatively rare and its coexistence with other primary non-breast cancers exceptional. Here, we report the case of a 50-year-old man who presented with symptoms of rectal adenocarcinoma and in whom a synchronous, asymptomatic cancer of the left breast was found incidentally at physical examination.
\end{abstract}

\section{Introduction}

Male breast cancer is relatively rare, accounting for about $1 \%$ of all breast cancer cases with an incidence of approximately 1 per 100,000 men per year [1]. Genetic, hormonal and environmental factors involved in female breast carcinogenesis also play a role in the etiology of male breast cancer [2]. Women with breast cancer have an increased risk of developing a second, breast or non-breast, malignancy and the same appears to apply to male breast cancer patients [3]. Here, we report a rare case of synchronous presentation of breast and rectal cancer in a man.

\section{Case Report}

A 50-year-old man presented with a one-month history of rectal bleeding, chronic constipation, weight loss of $10 \mathrm{~kg}$ over the last 3 months but no appetite loss. He had arterial hypertension treated with furosemide, but otherwise his past medical history and family history were unremarkable. Physical examination revealed a fit man. His body weight was $100 \mathrm{~kg}$ and the body mass index was $31.21 \mathrm{~kg} / \mathrm{m}^{2}$. His abdomen was soft, with normal bowel sounds, no distension, and no palpable masses. Rectal examination revealed an easily bleeding, $4-\mathrm{cm}$ polypoid mass confined to the anterior wall, about $6 \mathrm{~cm}$

Anastasios J. Karayiannakis, MD, $\mathrm{MSc}, \mathrm{PhD}$
Second Department of Surgery Democritus University of Thrace, Medical School GR-68 100 Alexandroupolis (Greece)

Tel. +30 25510 74006, E-Mail akarayan@usa.net 
from the anal verge. Routine physical examination of the breast revealed an asymptomatic, painless, well-circumscribed, hard but mobile nodule of $3 \mathrm{~cm}$ in diameter below the left nipple. The contralateral breast was normal and there was no evident gynecomastia. Supraclavicular or axillary lymph nodes were not palpable. Laboratory tests, complete blood count, blood biochemistry and tumor markers (CEA, CA19-9 and CA15-3) were all within normal limits. Total flexible colonoscopy confirmed the presence of a rectal tumor whose biopsy revealed a well-differentiated adenocarcinoma. There were no other lesions in the entire colon. Mammography and computed tomography suggested carcinoma of the left breast and this was confirmed by fine needle aspiration biopsy (fig. 1, fig. 2). Abdominal and thoracic computed tomography and whole body bone scintigraphy excluded distal metastases.

The patient underwent low anterior resection of the rectum along with total mastectomy and axillary lymph node dissection. Histological analysis revealed a well-differentiated adenocarcinoma of the rectum infiltrating the muscularis propria. There were no lymph node or distant metastases and the tumor was assigned as stage I (T2N0M0) according to the sixth edition of the TNM system (2002). Histological analysis of the breast tumor showed a poorly differentiated, histological grade III, not otherwise specified, infiltrating ductal carcinoma, measuring $3 \mathrm{~cm}$ in diameter. Ductal carcinoma in situ components of cribriform and comedo type were also detected. Immunostaining for both estrogen and progesterone receptors showed strong positivity whilst the expression of c-erbB-2 (Her-2/neu) was negative. There was no evidence of metastatic disease in the axillary lymph nodes and therefore, the tumor was staged as IIA (T2N0M0) according to the TNM system of the International Union against Cancer. The genetic testing for BRCA2 mutation showed no abnormality.

The case was presented to the Tumor Board of our institution and the decision was made to administer systemic chemotherapy followed by hormonotherapy for the breast cancer. This decision was based on the size of the tumor (3-cm lesion) and its poor differentiation. Adjuvant treatment for the rectal cancer was not recommended because both chemotherapy and radiotherapy have not been shown to be of any benefit for stage I colorectal cancers. The patient started combination chemotherapy consisting of paclixatel at a dose of $175 \mathrm{mg} / \mathrm{m}^{2}$ plus capecitabine at a dose of $2,000 / \mathrm{m}^{2}$ orally for 14 consecutive days for six cycles repeated every 21 days. The treatment was well tolerated and at its completion, the patient was given oral anti-estrogen treatment (tamoxifen $20 \mathrm{mg}$ once daily for 5 years) and a standard follow-up was recommended. The last follow-up was 16 months after surgery and the patient remains clinically well, without evidence of locoregional or distant recurrence of either cancers.

\section{Discussion}

Breast cancer in men is uncommon. Genetic, hormonal and environmental factors are involved in its pathogenesis, with $15-20 \%$ of cases being hereditary, occurring mostly in men with germ-line mutations in the BRCA2 gene, whereas BRCA1 gene mutations are not associated with increased breast cancer susceptibility in men [2]. Risk factors like age, testicular disease, benign breast conditions, family history, Klinefelter's syndrome, liver cirrhosis, chest wall irradiation, hormonal treatment, obesity and alcohol consumption have also been implicated in male breast carcinogenesis. In our patient, no apparent risk factors were noted and the rectal tumor was assumed as sporadic rectal cancer. Our patient presented with an adenocarcinoma of the rectum, and an invasive ductal carcinoma of the left breast was diagnosed incidentally at the same time. Only one similar report has been detected in the literature where a synchronous breast cancer was found incidentally in a 72-year-old patient with sigmoid colon cancer [4]. Nevertheless, it is not clear which tumor was preceding the other one.

The risk of second malignancy among female breast cancer patients has been studied extensively, but very few studies have assessed the risk of second cancer, either breast or non-breast, among male breast cancer patients or the risk for developing breast cancer following any other primary cancer. Male patients had a 93-fold (95\% confidence interval (CI): 39.0-184) increased risk of developing a second breast cancer, the standardized 
incidence ratio (SIR) being highest (SIR: 111.7, 95\% CI: 10.5-410.9) in patients more than 10 years after the diagnosis of the primary breast cancer [5]. In a cohort of 404 men with breast cancer, $2.7 \%$ of them subsequently developed either synchronous or metachronous contralateral breast cancer, whereas $17 \%$ of them developed a second non-breast malignancy, mainly prostate, lung, colorectal and esophageal cancer [6]. Data from the California Cancer Registry on 1,926 men with a first primary breast cancer showed that $11.5 \%$ of them developed a second primary cancer. The risk of developing a second cancer was mostly elevated for breast cancer (SIR: 52.12 , 95\% CI: 31.83-80.49), cutaneous melanoma (SIR: 2.98, 95\% CI: 1.63-5.00) and gastric cancer (SIR: 2.11, 95\% CI: 1.013.88 ), and there was a tendency towards higher risks of second malignancies among younger men compared to older men with the risk increasing over time [7]. Conversely, data from 1,788 men with breast cancer registered with the Surveillance, Epidemiology and End Results (SEER) Program showed no increase in the overall subsequent nonbreast cancer risk [8]. However, a 30-fold increased risk (SIR: 29.64, 95\% CI: 15-52) of developing subsequent contralateral breast cancer was found. The risk was much higher (SIR: 110.29, 95\% CI: 13.33-401.32) for those diagnosed with their primary breast cancer before the age of 50 years [8]. Pooled data on 3,409 men with primary breast cancer from 13 large cancer registries showed that $12.5 \%$ of patients subsequently developed a second cancer other than breast cancer [9]. The risk was higher for cancers of the small intestine, pancreas or prostate, myeloid leukemia, non-melanoma skin cancer, and cancer of the lymphohematopoietic system. Data from 225 male breast cancers detected after any other primary neoplasm showed that the risk of developing breast cancer was higher after lymphohematopoietic neoplasms (SIR: 1.80, 95\% CI: 1.07-2.84), mainly multiple myeloma (SIR: 3.77, 95\% CI: 1.03-9.65), and after prostate cancer (SIR: 1.19, 95\% CI: $0.90-1.54$ ), mostly $1-9$ years after prostate cancer diagnosis (SIR: 1.45 , 95\% CI: $1.07-$ 1.92). The risk of subsequent colorectal (SIR: 1.35, 95\% CI: 1.02-1.76) or rectal (SIR: 1.78, 95\% CI: 1.20-2.54) cancer development was elevated, occurring mostly in older patients (diagnostic age for breast cancer over 75 years) after 1-9 years of follow-up.

A number of population-based studies evaluated the association between breast and colorectal cancer in the reverse direction, namely the risk of second primary noncolorectal cancer occurring after colorectal adenocarcinoma [10-15]. The incidence of synchronous or metachronous non-colorectal cancer was $2-6 \%$. The most common malignancies seen were cancer of the stomach, especially in countries with a high incidence of gastric cancer, intestinal, kidney, prostate, uterine, cervical and ovarian cancer [10-15]. The majority of those secondary malignancies developed within 3 years after the primary colorectal cancer, although some developed 5 years or more after the initial diagnosis of colorectal cancer. Regarding breast cancer, Ueno et al. [10] reported that when colon cancer was associated with other cancers in women, the most frequently encountered neoplasm was gastric cancer $(1.4 \%)$ followed by breast cancer $(0.4 \%)$. Similar results were reported by Kan et al. [12]. An increased risk of developing breast cancer in women who survived colorectal cancer was also reported [11]. However, Evans et al. [14], after evaluating 61,433 men and 65,848 women with cancer included in the Thames Cancer Registry, failed to detect an increased risk for breast cancer development after diagnosis of colorectal cancer in women and there were no cases of breast cancer in the male cohort. When data on 262,600 cases of colorectal cancer from the SEER Program were assessed for the occurrence of subsequent primary cancers, it was found that the significantly increased risk of developing breast cancer after colorectal cancer in women was valid only for cancers reported during the first 5 months of follow-up (suggesting 
synchronous lesions), whereas the risk of metachronous cancer was not elevated and again no cases of male breast cancer were found [15].

Differences among cancer registries regarding cohort characteristics, disease reporting, synchronous or metachronous definition, length of follow-up and coding policy may account at least in part for the variation in results. Apparently, multifocal cancers may occur; however, an elevated risk of synchronous cancers among persons with colorectal carcinoma could be attributed to 'lead time bias' since second tumors might be detected before becoming clinically apparent because of increased medical scrutiny. In addition, when two cancers are diagnosed at the same time, the primary cancer may be defined arbitrarily as in our patient, who presented with symptoms from the rectal cancer, whereas the breast cancer was diagnosed incidentally during routine clinical examination. In this patient, the association between a frequent cancer-like rectal cancer and breast cancer cannot be explained by genetic alterations, environmental or other risk factors, and mechanisms of carcinogenesis shared by these two cancers. Data from the aforementioned population studies precluded a bidirectional association between breast and colorectal cancer, and a causal relationship between them cannot be demonstrated. A modestly increased risk of subsequent second colonic or rectal cancer was found in older males with breast cancer in the long-term follow-up but not vice versa, implying different etiologies for these two cancers. The synchronous rectal cancer in our patient was probably sporadic (since there was no polyposis of the large bowel and his family history for colorectal or other cancers was negative) and would have occurred in the absence of breast cancer. Although there were no BRCA2 mutations, alterations in the expression and function of other as yet unknown genes can not be excluded. In this context, the frame-shift mutation 1100delC in the cell cycle checkpoint kinase 2 gene (CHEK2) has been reported to be a low-penetrance breast cancer-predisposing gene associated with a three- to five-fold increased risk of breast cancer [16]. Recent studies have shown that the $1100 \mathrm{delC}$ variant of CHEK2 confers also a colorectal cancer risk in hereditary nonpolyposis colorectal cancer (HNPCC) and HNPCC-related families [17, 18]. These findings suggest that CHEK2 is a multiorgan cancer susceptibility gene acting probably in synergy with other susceptibility genes. Its function as a 'breast-colon cancer' gene, either by conferring a high risk of one cancer type and a slightly increased risk of the other or through a predisposition to one of the two cancers and chance occurrence of the other, may explain the simultaneous occurrence of breast and colorectal cancer in 'breast-colon cancer' patients [19]. When two primary cancers are diagnosed simultaneously, the systemic treatment should be directed against the tumor with worst prognosis. In our patient, systemic chemotherapy for breast cancer was administered because of the more advanced stage compared to the rectal cancer. Adjuvant treatment for the rectal cancer was not given because neither chemotherapy nor radiotherapy has shown any benefit for stage I colorectal cancer.

We conclude that male breast cancer patients may develop, either synchronously or metachronously, a second non-breast primary cancer such as colorectal cancer, and this possibility should be considered during primary evaluation and long-term follow-up. 


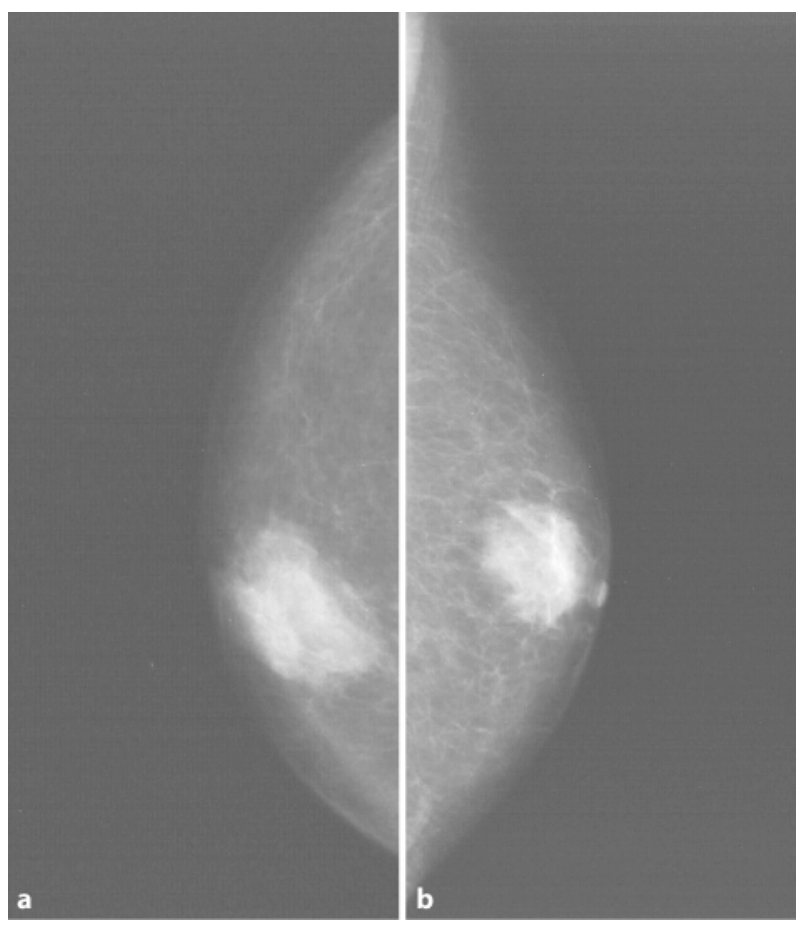

Fig. 1. Mammography of the left breast revealed a 3-cm nodule.

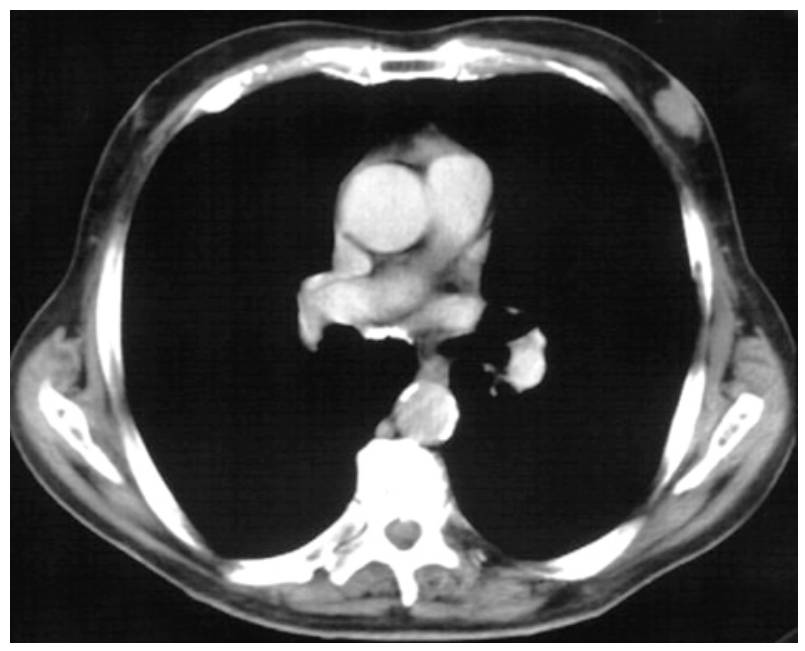

Fig. 2. Thoracic CT scan showing a mass in the left breast. 


\section{References}

1 Fentiman IS, Fourquet A, Hortobagyi GN: Male breast cancer. Lancet 2006;367:595-604.

2 Lynch HT, Watson P, Narod SA: The genetic epidemiology of male breast carcinoma. Cancer 1999;86:744-746.

3 Grenader T, Goldberg A, Shavit L: Second cancers in patients with male breast cancer: a literature review. J Cancer Surviv 2008;2:73-78.

$\checkmark 4$ Abboud B, Nassif J, Jaoude JB, Chahine G: Association synchrone d'un cancer du sigmoïde chez l'homme et d'un cancer du sein. Gastroenterol Clin Biol 2008;32:255-257.

5 Dong C, Hemminki K: Second primary breast cancer in men. Breast Cancer Res Treat 2001;66:171-172.

6 Cutuli BF, Lacroze M, Dilhuydy JM, Florentz P, Velten M, Allavena C, De Lafontan B, Resbeut M, Campana F, Graic $Y$, et al: Breast cancer in men: incidence and types of associated previous synchronous and metachronous cancers. Bull Cancer 1992;79:689-696.

7 Satram-Hoang S, Ziogas A, Anton-Culver H: Risk of second primary cancer in men with breast cancer. Breast Cancer Res 2007;9:R10.

8 Auvinen A, Curtis RE, Ron E: Risk of subsequent cancer following breast cancer in men. J Natl Cancer Inst 2002;94:1330-1332.

-9 Hemminki K, Scélo G, Boffetta P, Mellemkjaer L, Tracey E, Andersen A, Brewster DH, Pukkala E, McBride M, Kliewer EV, Chia KS, Pompe-Kirn V, Martos C, Jonasson JG, Li X, Brennan P: Second primary malignancies in patients with male breast cancer. Br J Cancer 2005;92:1288-1292.

10 Ueno M, Muto T, Oya M, Ota H, Azekura K, Yamaguchi T: Multiple primary cancer: an experience at the Cancer Institute Hospital with special reference to colorectal cancer. Int J Clin Oncol 2003;8:162-167.

11 Lee TK, Barringer M, Myers RT, Sterchi JM: Multiple primary carcinomas of the colon and associated extracolonic primary malignant tumors. Ann Surg 1982;195:501-507.

-12 Kan JY, Hsieh JS, Pan YS, Wang WM, Chen FM, Jan CM, Huang YS, Huang TJ, Wang JY: Clinical characteristics of patients with sporadic colorectal cancer and primary cancers of other organs. Kaohsiung J Med Sci 2006;22:547-553.

13 Lee WS, Lee JN, Choi S, Jung M, Baek JH, Lee WK: Multiple primary malignancies involving colorectal cancer - clinical characteristics and prognosis with reference to surveillance. Langenbecks Arch Surg 2010;395:359-364.

14 Evans HS, Møller H, Robinson D, Lewis CM, Bell CM, Hodgson SV: The risk of subsequent primary cancers after colorectal cancer in southeast England. Gut 2002;50:647-652.

15 Ahmed F, Goodman MT, Kosary C, Ruiz B, Wu XC, Chen VW, Correa CN: Excess risk of subsequent primary cancers among colorectal carcinoma survivors, 1975-2001. Cancer 2006;107(5 suppl):1162-1171.

16 Weischer M, Bojesen SE, Ellervik C, Tybjaerg-Hansen A, Nordestgaard BG: CHEK2*1100delC genotyping for clinical assessment of breast cancer risk: meta-analyses of 26,000 patient cases and 27,000 controls. J Clin Oncol 2008;26:542-548.

-17 Wasielewski M, Vasen H, Wijnen J, Hooning M, Dooijes D, Tops C, Klijn JG, Meijers-Heijboer H, Schutte M: CHEK2 1100delC is a susceptibility allele for HNPCC-related colorectal cancer. Clin Cancer Res 2008;14:4989_ 4994.

18 Meijers-Heijboer H, Wijnen J, Vasen H, Wasielewski M, Wagner A, Hollestelle A, Elstrodt F, van den Bos R, de Snoo A, Fat GT, Brekelmans C, Jagmohan S, Franken P, Verkuijlen P, van den Ouweland A, Chapman P, Tops C, Möslein G, Burn J, Lynch H, Klijn J, Fodde R, Schutte M: The CHEK2 1100delC mutation identifies families with a hereditary breast and colorectal cancer phenotype. Am J Hum Genet 2003;72:1308-1314.

19 Lipton L, Thomas HJ, Eeles RA, Houlston RS, Longmuir M, Davison R, Hodgson SV, Murday VA, Norbury CG, Taylor C, Tomlinson IP: Apparent Mendelian inheritance of breast and colorectal cancer: chance, genetic heterogeneity or a new gene? Fam Cancer 2001;1:189-195. 